\title{
Energy transfer from adipocytes to cancer cells in breast cancer
}

\author{
H. M. KIM", Y. K. LEE*, E. S. KIM, J. S. KOO* \\ Department of Pathology, Yonsei University College of Medicine, Seoul, South Korea \\ *Correspondence: kjs1976@yuhs.ac \\ ${ }^{\#}$ Contributed equally to this work.
}

Received October 17, 2019 / Accepted December 13, 2019

\begin{abstract}
Limitations of the current therapeutic approach have raised the need for a novel therapeutic agent in breast cancer. Recently, interest in drugs targeting the tumor microenvironment (TME) had drawn attention in the treatment of breast cancer. Furthermore, recent studies have suggested the role of adipocytes, which are part of the TME, in tumor initiation, growth, and metastasis. In this study, we investigated the metabolic interaction between adipocytes and breast cancer cells and its potential as a new therapeutic target in breast cancer. Breast cancer cell lines and human breast cancer tissue samples were evaluated. Compared to cancer cells cultured alone, or the control group, those co-cultured with adipocytes showed lipid transfer from adipocytes to cancer cells and it was different according to the molecular subtype of breast cancer. Breast cancer cells affected the lipolysis of adipocytes and adipocytes affected the $\beta$-oxidation of breast cancer cells. The key molecule of the process was fatty acid binding protein 4 (FABP4), which is combined with free fatty acid (FFA) and supports its migration to cancer cells. When FABP4 was suppressed, lipid transfer between adipocytes and cancer cells, lipolysis of adipocytes, and $\beta$-oxidation of breast cancer cells were reduced. Furthermore, the expression of lipid metabolism-related proteins and lipolysis-related proteins in breast cancer with adipose stroma showed significantly different expression according to the region of breast cancer tissue. Taken together, we demonstrated the metabolic interaction between adipocytes and breast cancer cells. Breast cancer cells increase the lipolysis in adipocytes and produce a fatty acid, and fatty acid enters into cancer cells. Also, adipocytes contribute to the survival and growth of cancer cells through increased mitochondrial $\beta$-oxidation by using fatty acid from adipocytes. The key molecule of the process is FABP4 and when FABP4 is suppressed, the metabolic interaction is reduced, suggesting its role as a potential therapeutic target.
\end{abstract}

Key words: adipocyte, breast cancer, energy transfer, lipid metabolism

Breast cancer is the most common type of cancer in women worldwide. Approximately 1.2 million cases of breast cancer are diagnosed annually. However, due to the large heterogeneity of the disease, no single established treatment yet exists. Even though the introduction of hormonal therapy based on the estrogen receptor (ER) or progesterone receptor (PR) status or target therapy according to the human epidermal growth factor type 2 (HER-2) status led to an advance in the treatment of breast cancer, up to $20 \%$ of patients eventually experience disease progression and even death [1]. Furthermore, triple negative breast cancer (TNBC), which shows negative ER, PR, and HER-2 expression is associated with extremely poor prognosis as effective targeted therapy is not available [2]. Limitations of the current therapeutic approach raised the needs for a novel therapeutic agent and recently, targeting the tumor microenvironment (TME), not the tumor itself, has evolved as a candidate of the therapeutic target. Unlike tumor cells, the TME includes the peritumoral element present in the region surrounding the tumor, immune system elements (such as macrophages and lymphocytes), blood vessel cells, fibroblasts, myofibroblasts, mesenchymal stem cells, adipocytes, and extracellular matrix.

In general, breast cancer is one of the tumors with various types of the stroma. Classifying breast cancer into two, one is fibrous stroma consisting of the extracellular matrix such as fibroblast and collagen, and the other is adipose stroma consisting of adipocytes. Normally, volume constituted by adipocytes in the entire breast is $7-56 \%$ and are accounted for $3.6-37.6 \%$ of the entire breast weight [3], and it accounts for the major component in breast cancer $[4,5]$. However, TME studies in breast cancer were mainly performed only in the fibrous stroma, especially cancer-associated fibroblast (CAF) [6-9]. It is known that cancer cells and stroma interact 
in diverse ways so the interaction between cancer cells and stroma in the respect of metabolism is well assumed. Reverse Warburg effect is one of the well-known theories explaining the metabolic interaction between cancer cells and fibrous stroma in breast cancer. Briefly, the theory assists that breast cancer cells produce reactive oxygen species such as a nitric oxide to place oxidative stress on stromal cells so that HIF-1a and $\mathrm{NF \kappa B}$ lead glycolysis, autophagy, and mitochondria dysfunction in stromal cells. Ketone bodies and lactates gained through the glycolysis in stromal cells enter cancer cells to efficiently produce adenosine triphosphate with oxidative phosphorylation in mitochondria, and in the end, contribute to the survival and growth of cancer cells. The theory exemplifies such stromal cells with CAF without the expression of caveolin-1 [10-13].

However, not only CAF but also the role of adipocyte in tumor initiation, growth, and metastasis has been reported. The role of adipocyte in tumorigenesis is referred as "adiponcosis" [14], and in a study of ovary cancer, lipolysis in adipocytes, and mitochondrial $\beta$-oxidation in cancer cells by the lipid transfer and the interaction between ovary cancer cells and omental adipocytes were reported in ovary cancer with metastasis to omentum [15]. In short, free fatty acid (FFA) is released from the nearby omental adipocytes and it is uptaken by metastatic cancer cells to become a substrate of mitochondrial $\beta$-oxidation. Based on the finding of this study, in vitro and in vivo studies in breast cancer showed that adipocyte enhances tumor growth [16, 17] or cancer cell proliferation $[17,18]$. Furthermore, one study showed by using a breast cancer cell line MCF-7, that insulin like growth factor binding protein 2 secretion by adipocyte stimulates breast cancer invasion [19]. Compared to the reverse Warburg effect between breast cancer and fibrous stroma, this theory insists that the stromal cells provide another type of energy, FFA in this case, for the growth of cancer cells [15]. Reverse Warburg effect well explains the metabolic interaction between breast cancer and fibrous stroma. However, the interaction between breast cancer and adipose stroma, which is the other important component of breast cancer stroma, is not yet studied sufficiently. Therefore, in this study, we investigated the metabolic interaction between adipocytes and breast cancer cells and its potential as a new therapeutic target in breast cancer.

\section{Materials and methods}

Culture and treatment of breast cancer cell lines. MDA-MB-453 (HTB-131), MDA-MB-435S (HTB-129), MDA-MB-231 (CRM-HTB-26), MDA-MB-468 (30-4500K), and MCF-7 (HTB-22) cells were purchased from the American Type Culture Collection (ATCC, Rockville, MD, USA). The general information about breast cancer cell lines used in this study is shown in Table S1. MDA-MB453, MDA-MB-435S, MDA-MB-231, and MDA-MB-468 cells were grown in Dulbecco's modified Eagle's medium
(DMEM) supplemented with 10\% FBS, 1\% penicillin-streptomycin (Hyclone) in a humidified of $5 \% \mathrm{CO}_{2}$ atmosphere. MCF-7 cells were cultured in DMEM with no phenol red (Life Technology, Grand Island, NY, USA) supplemented with $10 \% \mathrm{FBS}, 1 \%$ penicillin-streptomycin, and $0.1 \mathrm{mg} / \mathrm{ml}$ insulin.

Human breast cancer tissue selection. We included only those patients diagnosed with invasive breast cancer and underwent breast cancer surgery at Severance Hospital. Patients who received hormonal therapy or chemotherapy prior to surgery were excluded. This study was approved by the Institutional Review Board of Yonsei University Severance Hospital. All slides were retrospectively reviewed by a breast pathologist (Koo JS) and histological evaluation was conducted by hematoxylin and eosin ( $\mathrm{H} \& \mathrm{E})$ stained slides. The histological grade was assessed using the Nottingham grading system. Clinicopathological parameters evaluated in each breast cancer included patient age at initial diagnosis, lymph node metastasis, tumor recurrence, distant metastasis, and patient's survival.

Differentiation of 3T3-L1 cells to mature adipocytes. 3T3-L1 preadipocytes were purchased from the American Type Culture Collection (ATCC, Rockville, MD, USA; Cat no. CL-173). Cells were maintained in DMEM containing $10 \%$ fetal calf serum (Life Technology) until adipocyte differentiation. 3T3-L1 preadipocyte cell line was differentiated as previously reported [20]. To confirm mature adipocyte differentiation, Oil Red $\mathrm{O}$ staining was conducted, and immunoblotting was performed with antibodies for adipogenesis markers. Fatty acid binding protein 4 (FABP4) inhibitor (BMS309403) was purchased from Cayman Chemical and in the experiments used at $30 \mathrm{mM}$ for the indicated time.

Co-culture of breast cancer cells with mature adipocytes. Breast cancer cells and mature adipocytes were co-cultured using Costar Transwell culture vessels $(0.4 \mathrm{~mm}$ pore size, Corning, NY, USA) for the indicated time. Breast cancer cells were seeded in the upper chamber and co-cultured with or without mature adipocytes at day 10 after the induction of differentiation in the lower chamber.

Cell proliferation test. To test cell proliferation, breast cancer cell lines were co-cultured with or without mature adipocytes for $48 \mathrm{~h}$, trypsinized, and stained with $0.4 \%$ Trypan blue stain solution (Life Technology). Viable cells were counted using a hemocytometer by a least three indifferent experiments.

Immunocytochemistry and confocal microscopy. Breast cancer cells were seeded on the coverslips and cultured with or without mature adipocytes. After 2 days of co-culture, cells were fixed with $4 \%$ paraformaldehyde for $20 \mathrm{~min}$ and permeabilized with $0.2 \%$ Triton $\mathrm{X}-100$ for $10 \mathrm{~min}$ at room temperature, then washed with PBS. The cells were incubated in blocking solution (5\% normal donkey serum in PBS) for $1 \mathrm{~h}$ and then incubated with the primary antibody in PBS for $1 \mathrm{~h} 30 \mathrm{~min}$ at room temperature. After washing with PBS-T (PBS containing $0.1 \%$ Tween-20), the cells were incubated 
with a second antibody conjugated with a fluorescent dye for $1 \mathrm{~h}$ at room temperature. The cells were counterstained with Hoechst 33342 (Invitrogen, Carlsbad, CA, USA) and mounted on slides with mounting medium (Dako, Glostrup, Denmark). Images were acquired under an LSM 700 META confocal laser scanning microscope equipped with epifluorescence and a digital image analyzer (Carl Zeiss, Zena, Germany). Z-stacked images were acquired at 400× magnification.

Quantitative real-time polymerase chain reaction (qRT-PCR). Total RNA of cultured cells was isolated using the RNeasy Mini Kit according to the manufacturer's instructions (Qiagen, Valencia, CA). Real-time PCR was performed using the One Step SYBR PrimeScript ${ }^{\text {tim }}$ RT-PCR Kit (Takara Shuzo Co, Japan) according to the manufacturer's instructions. The relative mRNA expression levels of target gene were normalized to the housekeeping gene GAPDH (glyceraldehyde 3-phosphate dehydrogenase). All primers of the target genes were designed using Primer 3 software. Primer sequences are described in Table S2.

Immunoblotting. The cells were harvested and lysed in RIPA buffer (50 mM Tris- $\mathrm{HCl}$ pH 7.4, 1\% NP-40, 0.25\% $\mathrm{Na}$ deoxycholate, $150 \mathrm{mM} \mathrm{NaCl}, 1 \mathrm{mM}$ EDTA, 0.1\% SDS) containing protease inhibitors. Cellular lysates were centrifuged at $13,000 \times \mathrm{g}$ for $15 \mathrm{~min}$ at $4^{\circ} \mathrm{C}$. The protein concentration of each cell lysate was measured via the BCA assay (Thermo-Scientific), and an equal amount of protein from each sample extract was separated on SDS-PAGE gels and blotted onto nitrocellulose membranes (Bio-Rad). Western blots were performed as previously described [21], and specific bands were detected using the ECL solution kit (GE Healthcare Life Sciences).

Tissue microarray. On H\&E-stained slides of cancers, a representative area was selected, and a corresponding spot was marked on the surface of the paraffin block. Using a biopsy needle, the selected area was punched out and a $3 \mathrm{~mm}$ tissue core was placed into a $6 \times 5$ recipient block. The tissue of invasive cancer was extracted. Two tissue cores were extracted to minimize extraction bias. Each tissue core was assigned with a unique tissue microarray location number that was linked to a database containing other clinicopathologic data.

Immunohistochemistry. The antibodies used for immunohistochemistry (IHC) in this study are shown in Table S3. All IHC staining was conducted using FFPE tissue sections. Briefly, $5 \mu \mathrm{m}$-thick sections were obtained with a microtome, transferred into adhesive slides, and dried at $62{ }^{\circ} \mathrm{C}$ for 30 minutes. After incubation with primary antibodies, immunodetection was performed with biotinylated antimouse immunoglobulin, followed by peroxidaselabeled streptavidin using a labeled streptavidin biotin kit with 3,3'-diaminobenzidine chromogen as substrate. The primary antibody incubation step was omitted in the negative control. Slides were counterstained with Harris hematoxylin. All IHC markers were accessed by light microscopy.
Protein extraction from formalin-fixed, paraffinembedded tissues, and western blot. Protein extractions from FFPE tissues were performed using the Qproteome FFPE tissue kit (Qiagen, Hilden, Germany). Briefly, two or three sections from the same block were deparaffinized in xylene and rehydrated in graded series of alcohol. The tissues were mixed with FFPE extraction buffer containing $\beta$-mercaptoethanol, incubated at $100^{\circ} \mathrm{C}$ for $20 \mathrm{~min}$, at $80^{\circ} \mathrm{C}$ for $2 \mathrm{~h}$ with agitation at $750 \mathrm{rpm}$ and then centrifuged for $15 \mathrm{~min}$ at $14,000 \times \mathrm{g}$ at $4^{\circ} \mathrm{C}$. The supernatant containing the extracted proteins were determined by the Bradford assay (Bio-Rad Laboratories, Hercules, CA). An equal amount of protein from each sample extract was separated on SDS-PAGE gels and blotted onto nitrocellulose membranes (Bio-Rad). Western blotting was performed with primary antibodies against PLIN1 (perilipin-1), CPT1A (carnitine palmitoyltransferase 1A), HSL (hormone-sensitive lipase), FABP4, FAS (fatty acid synthase), and $\beta$-actin (Abcam, Cambridge, UK), and specific bands were detected using the enhanced chemiluminescence kit (GE Healthcare Life Sciences, Little Chalfont, UK).

\section{Results}

Selection and induction of differentiation of adipocytes, which would be co-cultured with breast cancer cells. The adipocytes, which would be co-cultured with breast cancer cells, were classified into two groups: primary adipocyte (RM, reduction mammoplasty; TU, tumorectomy) and adipocyte cell line (3T3-L1, 3T3-F442A), and cultured. As a result, the 3T3-L1 adipocyte cell line was selected on account of the relatively long period of subculture and good reproducibility. Three to 14 days after the induction of 3T3-L1 differentiation by treatment with insulin, dexamethasone, and 3-isobutyl-1methylxanthine, the differentiation to mature adipocyte was confirmed with Oil Red O staining and western blotting. In this study, adipocytes were used 10 days after the induction of differentiation.

Lipid transfer from adipocytes to cancer cells according to the breast cancer molecular subtypes. In this study, breast cancer cells were co-cultured with adipocytes by an indirect method using a Transwell. Lipid transfer from adipocytes to cancer cells according to the molecular subtypes of breast cancer was analyzed by Bodipy 493/503, which stains neutral fat. Compared to cancer cells cultured alone, or the control group, those co-cultured with adipocytes produced more neutral fat (Figure 1A). Compare to the control group, MDA-MB-231 cells about 12 times or more, MDA-MB-435S cells about 4-5 times or more, and MCF-7 and MDA-MB453 cells about 2-3.6 times or more frequently showed lipid transfer. Therefore, the more frequent lipid transfer takes place in triple negative breast cancer (TNBC) cells (Figure 1B). Furthermore, cell growth was faster when cancer cells co-cultured with adipocytes than when cultured alone (Figure 1C). 
A
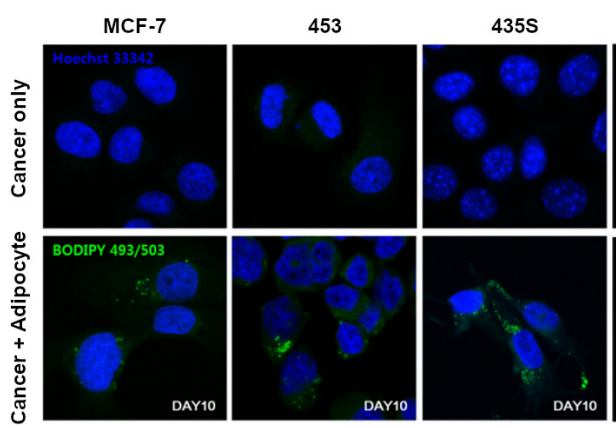

C

B

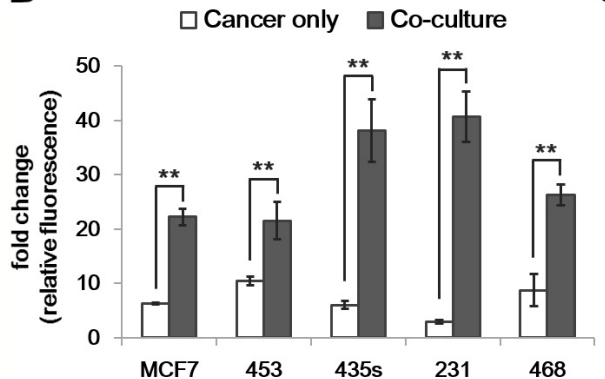

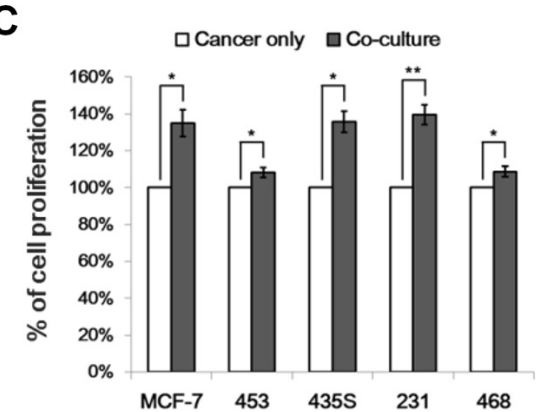

Figure 1. A, B) Co-culture of breast cancer cells with mature adipocytes induces lipid transfer into breast cancer cells. Breast cancer cells were grown on coverslips and co-cultivated with or without mature adipocytes (day 10) for $48 \mathrm{~h}$. The cells were fixed and stained with BODIPY $493 / 503$ (green). Images were acquired under a confocal microscope (A) and relative fluorescence was quantified by ImageJ (B), scale bar: 20 m. C) Five subtypes of breast cancer cells were co-cultured with mature adipocytes for $\mathbf{4 8} \mathrm{h}$ and stained with Trypan blue. Viable cells were counted using a hemocytometer. Bars represent the mean \pm S.D. of three independent experiments, ${ }^{* *} \mathrm{p}<0.01,{ }^{*} \mathrm{p}<0.05$.

The origin of lipids located in breast cancer cells. After the uptake of fluorescent dodecanoic acid analog in adipocytes using Fatty acid uptake assay kit (Molecular Device, CA, USA), adipocytes with fluorescently labeled lipids were co-cultured with breast cancer cells. We took the fluorescent image from cancer cells by a confocal microscope. As a result, it was confirmed that the fluorescent signal increased in cancer cells (Figure S1A). The signal from 4 cells excluding MDA-MB-453 cells was highly increased about 5-11 times, and that from MDA-MB-231 cells proved the largest augmentation (11.26 times) similar to that in lipid transfer (Figure S1B).

The effect of breast cancer cells on the lipolysis of adipocytes according to the breast cancer molecular subtypes. In the results shown above, FFAs derived from adipocytes were increased when breast cancer cells were co-cultured with adipocytes. It suggests the possibility that triglycerides stored in adipocytes were hydrolyzed into FFAs and glycerol. Therefore, we compared the amount of FFAs and glycerol with the Lipolysis assay kit (Zenbio, NC, USA) of two groups: adipocytes cultured alone (the control group) and adipocytes separated after co-culture with breast cancer cells according to the molecular subtypes. As a result, the amount of FFAs and glycerol was higher in the group co-cultured cancer cells with adipocytes than that of each group cultured cancer cells alone or adipocytes alone (Figures 2A, 2B).
For the lipolysis in adipocytes to take place, the activation of $\beta$-adrenergic receptor stimulation and $G$ proteincoupled cascade leading the phosphorylation of HSL and PLIN1, and consequent hydrolysis of triglyceride is necessary. Therefore, we analyzed the difference of phosphorylation of HSL between adipocytes cultured alone and of those separated after co-culture with breast cancer cells. Consequently, it was confirmed that a significant increase in the phosphorylation of HSL is shown in adipocytes co-cultured with cancer cells from cell lines except for MDA-MB-453 cells (Figures 2C, 2D).

The effect of adipocytes on the $\beta$-oxidation of breast cancer cells. Because the lipolysis in adipocytes was increased compared to that in adipocytes co-cultured with breast cancer cells, we investigated the metabolic changes in cancer cells. AMP-activated protein kinase (AMPK) is a kinase playing the role of a switch of the main metabolism. When AMPK is phosphorylated, the phosphorylation of acetyl-CoA carboxylase as the sub-step of AMPK phosphorylation takes place. As a result, CPT1a transports the fatty acid into the mitochondria, and $\beta$-oxidation will occur. Thus, we compared AMPK phosphorylation of two groups (breast cancer cells cultured alone, and breast cancer cells separated after co-culture with adipocytes) to analyze the effect of adipocytes on $\beta$-oxidation of breast cancer cells. It was confirmed that AMPK phosphorylation was signifi- 
A

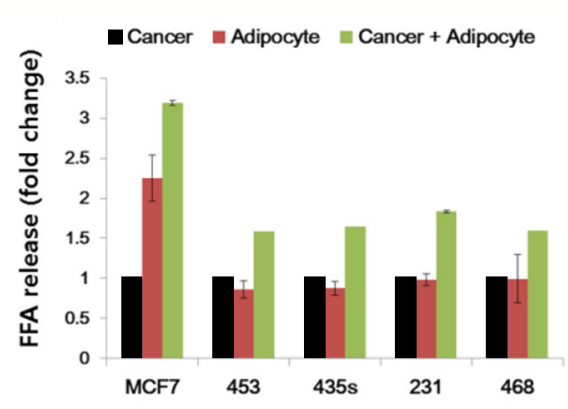

C

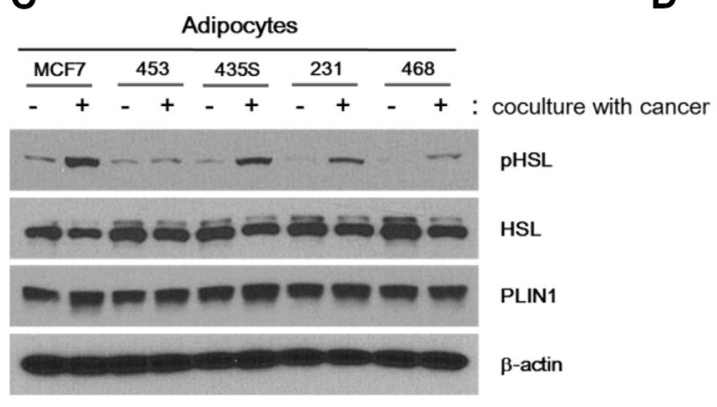

B

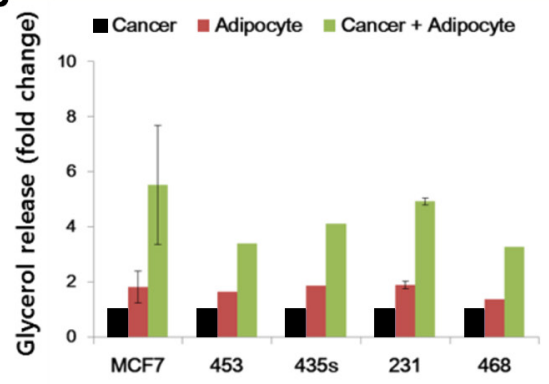

D

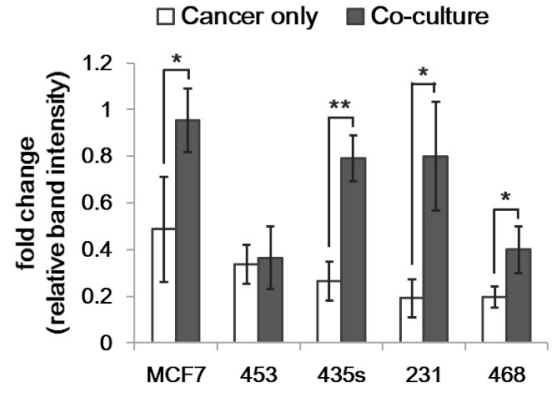

Figure 2. The co-culture of breast cancer cells with mature adipocytes enhances lipolysis in adipocytes. A) Free fatty acids and B) glycerol were detected by lipolysis assay in mature adipocytes. C) Adipocytes were co-cultured with or without breast cancer cells for $48 \mathrm{~h}$ and then performed by immunoblotting with the indicated antibodies. D) Phosphorylated HSL (pHSL, Ser660) was quantified by ImageJ. Columns represent the mean \pm S.D. of three independent experiments, ${ }^{* *} \mathrm{p}<0.01,{ }^{*} \mathrm{p}<0.05$.

cantly increased in cancer cells co-cultured with adipocytes (Figures 3A, 3B). In addition, the difference of CPT1a and acyl-CoA oxidase 1 (ACOX1) protein expression level in cancer cells co-cultured with adipocytes was investigated with western blotting. As a result, CPT1a protein expression level was evidently increased in MDA-MB-435S cells. However, the level was not increased in other breast cancer cell lines and ACOX1 protein expression level showed no significant difference in cancer cells co-cultured with adipocytes compared to that in the control group (Figure 3C). In consequence, we analyzed CPT1a and ACOX1 mRNA expression level and identified that CPT1a and ACOX1 mRNA expression level, which showed an increase in MDA-MB-435S and MDA-MB-231 cells co-cultured with adipocytes (Figure 3D). In MCF-7 cells, the mRNA and protein expression level of CPT1a and mRNA was not correlated to the presence of co-cultured adipocytes, but there was a notable different staining pattern with confocal microscopy. When cancer cells cultured alone, CPT1a was distributed over the whole cell. On the other hand, it was accumulated in a specific area in the cancer cells co-cultured with adipocytes. Also, ACOX1 showed a similar pattern with CPT1a and the fluorescence signal was increased in cancer cells co-cultured with adipocytes. In MDA-MB-435S cells, CPT1a was accumulated in a specific area within the cells (Figure 3E).
Co-culture of adipocyte and normal breast cell line. To confirm that these results were specific to the breast cancer cell line, we co-cultured a normal breast cell line, MCF10A mammary epithelial cell line with adipocytes. Afterward, lipid transfer and $\beta$-oxidation were evaluated by isolating MCF10A. Lipid transfer was not noticeable in MCF10A co-cultured with adipocytes as well as MCF10A cultured alone (Figure S2A). In addition, MCF10A showed no changes in the expression of CPT1a, ACOX1, and pAMPK regardless of co-culture with adipocytes (Figure S2B), showing that adipocytes do not affect $\beta$-oxidation of a normal breast cell line, MCF10A. These findings suggest that the metabolic interaction between adipocytes and breast cancer cells is specific to breast cancer line, and in further experiments, the MCF10A cell line was used as a negative control.

The expression of FABP4 in breast cancer cell lines. The expression of FABP4 in various breast cancer cell lines was examined. As a result, all breast cancer cell lines showed higher expression in the case of cancer cells co-cultured with adipocytes than those cultured alone (Figures S3A, S3C). The FABP4 mRNA level was higher in cancer cells co-cultured with adipocytes than those cultured alone in MCF-7, MDA-MB-453, and MDA-MB-468 (Figure S3B).

Inhibition of FABP4 expression in adipocytes using FABP4 inhibitor. FABP4 inhibitor (BMS309403) was used to inhibit the expression of FABP4. Incubation of FABP4 inhib- 
A
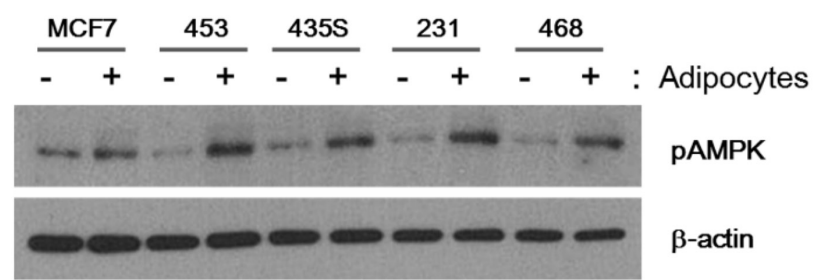

pAMPK

C
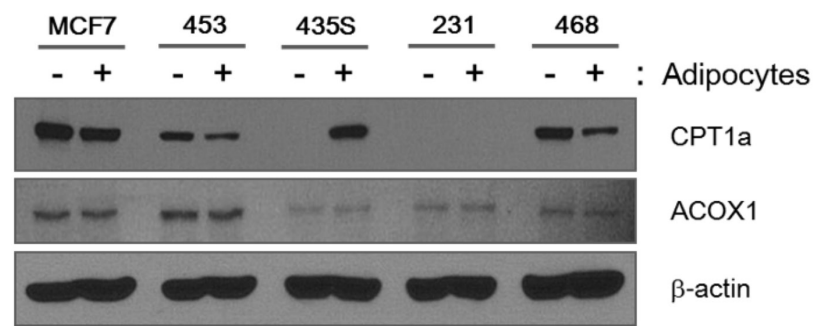

CPT1a

ACOX1

$\beta$-actin

D

$\square$ Alone $\square$ CPT1a $\square$ ACOX1

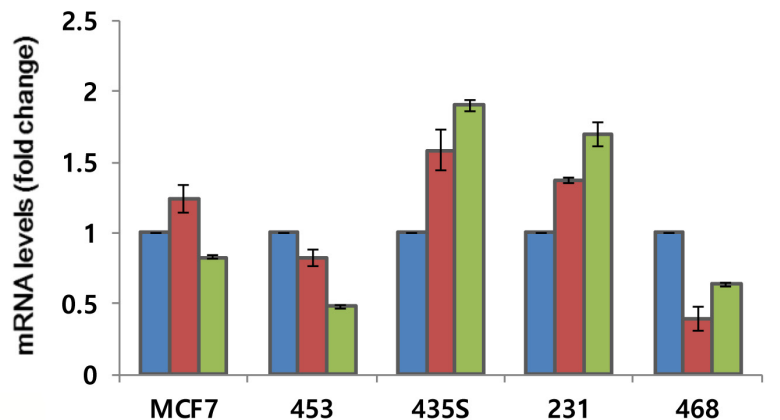

B

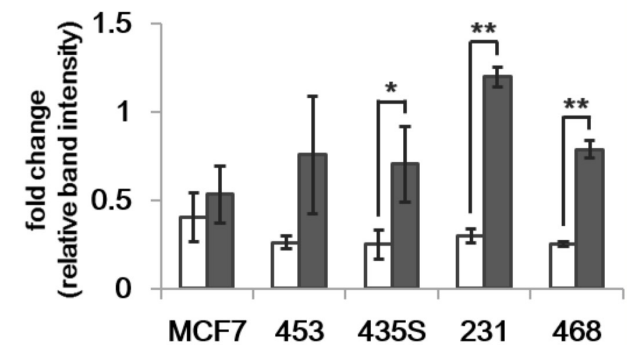

E

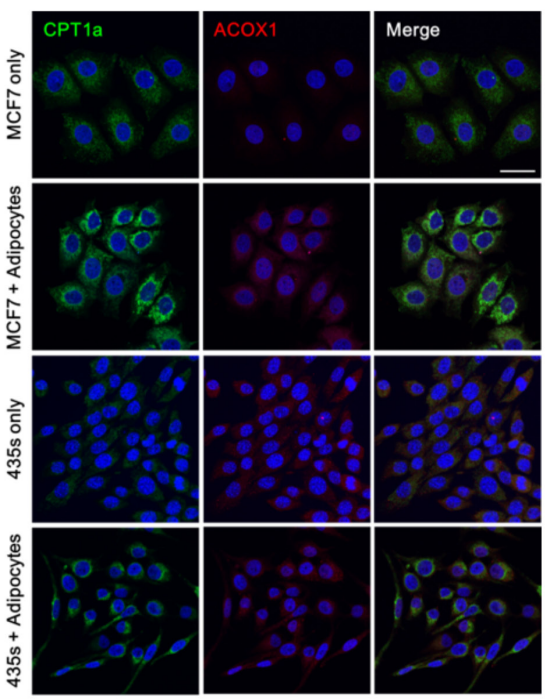

Figure 3. During co-culture, adipocytes activate $\beta$-oxidation in cancer cells. A, B) Five subtypes of breast cancer cells were co-cultured with mature adipocytes for $48 \mathrm{~h}$. Phosphorylation of AMPK was determined by immunoblotting (A) and relative band intensity was quantified by ImageJ (B). C) The expression of CPT1a and ACOX1 was detected by immunoblotting. D) Relative mRNA expression of CPT1a and ACOX1 was analyzed by real-time PCR. E) Co-culture with adipocytes had concentrated expression of CPT1a and ACOX1 in MCF7 and increased CPT1a expression in MDA-MB-435s cells, scale bar: $100 \mu \mathrm{m}$. Columns represent the mean \pm S.D. of three independent experiments, ${ }^{* *} \mathrm{p}<0.01,{ }^{*} \mathrm{p}<0.05$.

itor during 3T3-L1 adipocyte differentiation was performed in different conditions (data not shown), and the expression of FABP4 was significantly inhibited when $30 \mathrm{mM}$ of FABP4 inhibitor was administered on day 6 (Figure S4A). Adipocyte that was used for co-culture in this study was mature adipocyte, which was on day 10 after differentiation. As FABP4 inhibitor, which was administered on day 6 should suppress FABP4 expression until day 12, we evaluated the expression of FABP4 on day 10, 11, 12, and 13 after administration of FABP4 on day 6. Our result showed that from day 10 to day 12, FABP4 and PLIN1 expression was reduced (Figure S4B), and Oil Red $\mathrm{O}$ staining also showed that lipid droplet within the adipocyte was significantly reduced (Figure S4C).

The effect of FABP4 inhibition on lipid transfer between adipocytes and cancer cells. The change of lipid transfer from adipocytes to cancer cells after the FABP4 inhibitor injection was studied. As a result, lipid transfer to all breast cancer cells except for normal breast cells was reduced in the case with FABP4 inhibitor injection than in the case without FABP4 inhibitor injection (Figures 4A, 4B).

The effect of FABP4 inhibition on lipolysis of adipocytes co-cultured with breast cancer cells. The effect of FABP4 inhibition on lipolysis of adipocytes co-cultured with breast cancer cells was examined. As a result, the amount of FFAs and glycerol was reduced in the case with FABP4 injection than in the case without FABP4 injection. The Lipolysis assay kit was used for the measurement (Figures 4C, 4D). The expression of pHSL, HSL, and PLIN1 was reduced in the case with FABP4 injection than in the case without FABP4 injection in adipocytes co-cultured with breast cancer cells (Figure 4E).

The effect of FABP4 inhibition on the $\boldsymbol{\beta}$-oxidation of breast cancer cells co-cultured with adipocytes. The effect of FABP 4 inhibition on the $\beta$-oxidation of breast cancer cells 
co-cultured with adipocytes was examined. As a result, the expression of pAMPK was reduced in the case with FABP4 injection than in the case without FABP4 injection in MDA-MB-435S and MDA-MB-231 (Figure 4F). The CPT1a mRNA level was reduced in the case with FABP4 injection than in the case without FABP4 injection in MDA-MB435S, MDA-MB-231, and MDA-MB-468 (Figure 4G). The ACOX1 mRNA level was reduced in the case with FABP4 injection than in the case without FABP4 injection in MCF-7, MDA-MB-435S, and MDA-MB-468 (Figure 4H).

The expression of lipid metabolism-related proteins in breast cancer with adipose stroma. The expression of lipid metabolism-related proteins in human breast cancer with adipose stroma was examined. The expression of ACOX1 and CPT1A was higher in cancer cells located within or in proximity to the adipose stroma rather than cancer cells located within the fibrous stroma. The expressions of FABP4 and HSL in adipocytes were different according to the location, which was higher in adipocytes located in the interface adjacent to cancer cells than in adipocytes located apart from cancer cells (Figure 5A).

The expression of lipolysis-related proteins in the different regions of breast cancer tissue. We investigated the expression of lipolysis-related proteins in the three different regions of breast cancer tissue, [central cancer with fibroblast (zone 1), cancer with adipocyte (zone 2), and adipocyte nearby cancer (zone 3)] (Figure 5B). The expression of PLIN1, HSL, and FABP4 were highest in zone 3, followed by zone 2 and 1, while CPT1A expression was highest in zone 2, followed by zone 3 and 1 (Figure 5C).

\section{Discussion}

In this study, we investigated the metabolic interaction between adipocytes and cancer cells according to molecular subtypes of breast cancer. It was further confirmed by an in

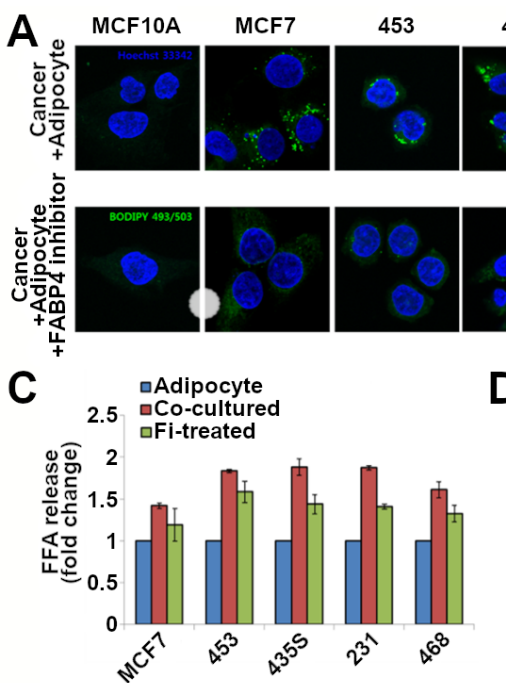

F

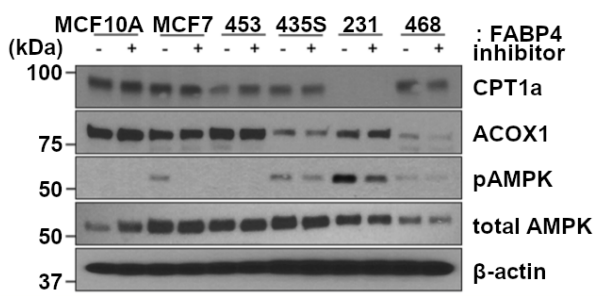

231
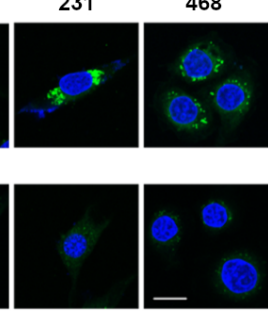

D $\quad$ Adipocyte

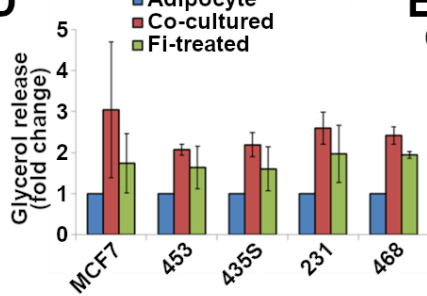

G

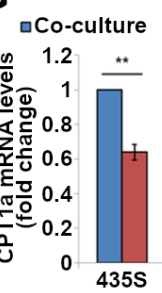

B

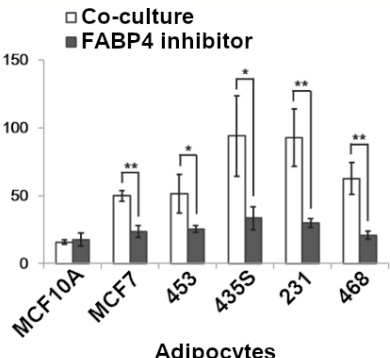

E MCF10A MCF7 $\underline{453} \underline{435 S \underline{231} \underline{468}}$

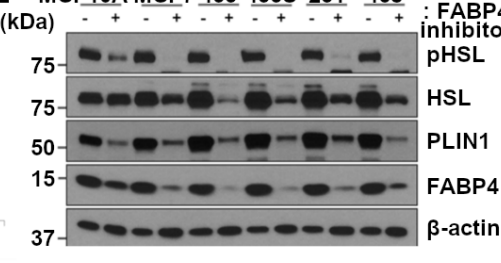

$\mathrm{H}$

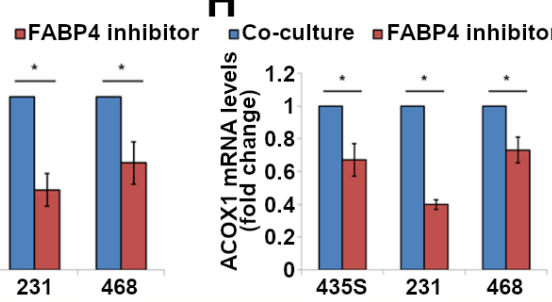

Figure 4. A, B) Co-culture of breast cancer cells with FABP4 inhibitor-treated adipocytes reduces lipid accumulation. Breast cancer cells were grown on coverslips and co-cultured with mature adipocytes or FABP4 inhibitor-treated adipocytes for $48 \mathrm{~h}$. Breast cancer cells were isolated and stained with BODIPY 493/503 (green). Images were acquired under a confocal microscope (A) and relative fluorescence was assessed by ImageJ (B), scale bar: $100 \mu \mathrm{m}$. Columns represent the mean \pm S.D. of three independent experiments, ${ }^{\star *} \mathrm{p}<0.01,{ }^{*} \mathrm{p}<0.05$. C-E) Co-culture of breast cancer cells with FABP4 inhibitor-treated adipocytes reduces lipolysis in adipocytes. Free fatty acids (C) and glycerol (D) were detected by lipolysis assay in mature adipocytes. E) The co-culture of breast cancer cells with mature adipocytes or FABP4 inhibitor-treated adipocytes for $48 \mathrm{~h}$, and then adipocytes were isolated and performed by immunoblotting with the indicated antibodies. Scale bar, $100 \mathrm{~mm}$. Bars represent the mean \pm S.D. of three independent experiments. F-H) FABP4 plays an important role in the interaction between breast cancer cells and adipocytes. Breast cancer cells were co-cultivated with mature adipocytes or FABP4 inhibitor-treated adipocytes for $48 \mathrm{~h}$. F) The cell lysates were subjected to SDS-PAGE and performed by immunoblotting with the indicated antibodies. G, H) Relative mRNA levels of CPT1a and ACOX1 were assessed by quantitative real-time PCR. mRNA expression was normalized to GAPDH and presented as fold change. Columns represent the mean \pm S.D. of three independent experiments, ${ }^{* *} \mathrm{p}<0.01,{ }^{*} \mathrm{p}<0.05$. 


\section{B}

A
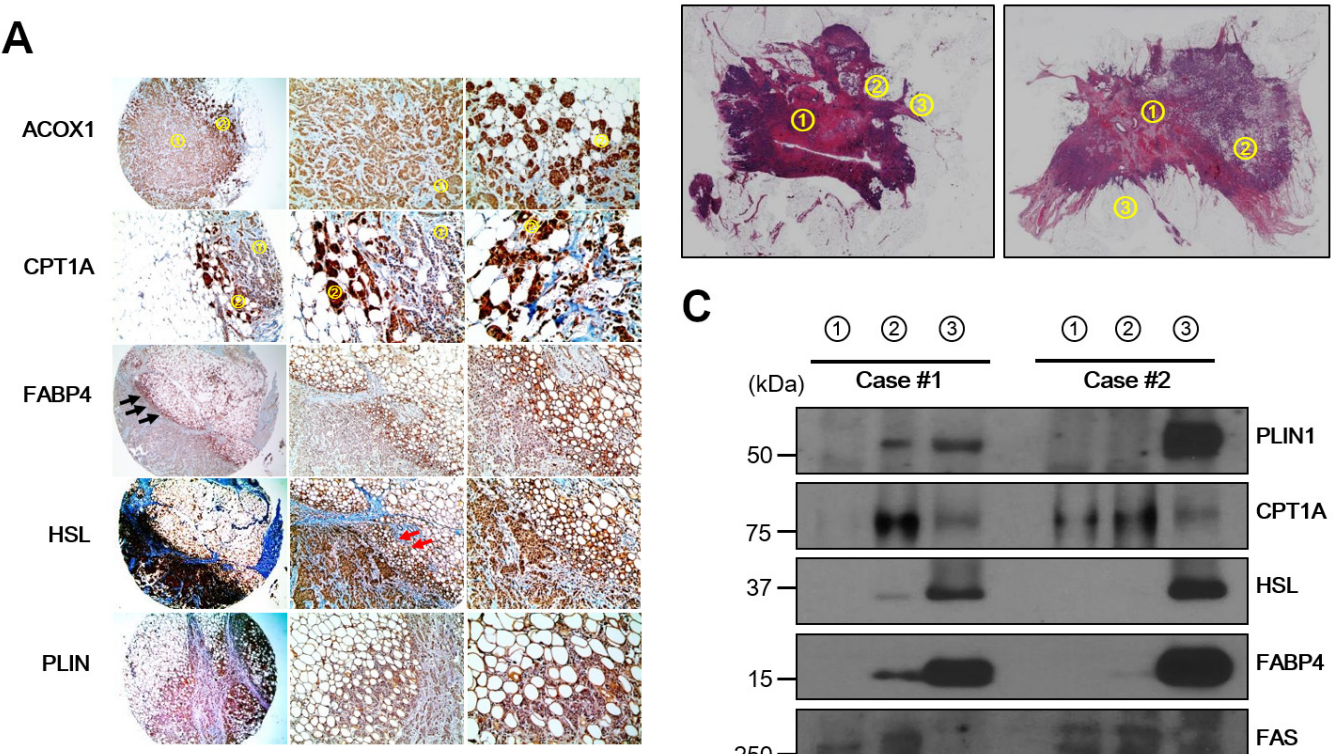

C

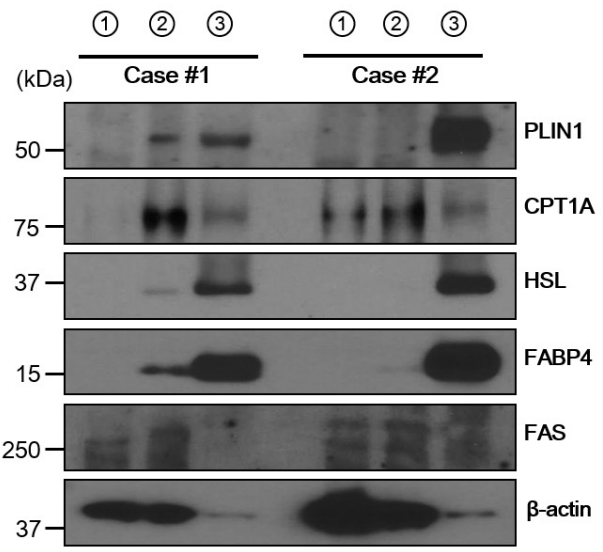

Figure 5. A) The expression of lipid metabolism-related proteins in breast cancer with adipose stroma. The expression of ACOX1 and CPT1A was higher in cancer cells located within or in proximity to the adipose stroma ( ) rather than cancer cells located within the fibrous stroma (,). The expressions of FABP4 and HSL in adipocytes were different according to the location, which was higher in adipocytes located in the interface adjacent to cancer cells (arrow) than in adipocytes located apart from cancer cells. B, C) The expression of lipolysis-related proteins in the different regions of breast cancer tissue. B) Formalin-fixed, paraffin-embedded breast cancer tissue samples were processed for H\&E staining to identify the target region. Central cancer with fibroblast, , Cancer with adipocyte, $f$ Adipocyte nearby cancer from two cases were selected. (H\&E, magnification $\times 12.5)$. $C)$ Protein extracts were isolated from FFPE breast cancer tissue samples and western blotting was performed with primary antibodies against PLIN1, CPT1A, HSL, FABP4, FAS, and $\beta$-actin.

vitro cell line study showing lipid transfer from adipocytes to breast cancer cells. This finding is in line with a previous study, which showed cytoplasmic lipid droplet accumulation in cancer cells when breast cancer cells and adipocytes are co-cultured [15]. Interestingly, this phenomenon was not observed when normal breast cells and adipocytes are co-cultured, suggesting that lipid transfer occurs specifically between adipocytes and cancer cells. In addition, the amount of lipid transfer was different according to the molecular subtypes of breast cancer cell line. Of note, the highest lipid transfer was observed in a TNBC type cell line, MDA-MB231. As TNBC is characterized as having aggressiveness and rapid proliferation, it could be reasonably speculated that lipid transfer from adipocytes is increased in TNBC. Since the proliferation was significantly increased in cancer cells when co-cultured with adipocytes than cultured alone, lipid transfer might play an important role in cancer cell proliferation. Accordingly, increased lipid transfer is expected in TNBC type with high proliferation ability. The pathways of these processes are outlined in Figure 6.

In addition to lipid transfer, we showed that adipocytes and breast cancer cells affect each other in another manner as well. We evaluated the metabolic interaction between adipo- cyte and breast cancer cells. As a result, breast cancer cells increased lipolysis in adipocytes, and adipocytes promoted the $\beta$-oxidation in breast cancer cells via increased AMPK phosphorylation. However, CPT1a and ACOX1, the enzymes involved in the mitochondrial $\beta$-oxidation, were increased in the TNBC type cell lines (MDA-MB-435S and MDA-MB231), although differences in mRNA and protein level were observed. Therefore, lipolysis in adipocytes and mitochondrial $\beta$-oxidation in cancer cells by the lipid transfer and the interaction between adipocytes and cancer cells were observed in breast cancer, especially in TNBC type. In MCF-7, although the mRNA and protein level of CPT1a and ACOX1 did not differ when it was co-cultured with adipocytes, the difference in expression pattern was shown. The expression was specifically accentuated in subcellular location. Further studies to find the association between this phenomenon and mitochondrial $\beta$-oxidation are required.

In a study by Nieman et al. which was performed on ovary cancer cells, lipolysis in adipocytes and mitochondrial $\beta$-oxidation in cancer cells by the lipid transfer, and the interaction between ovary cancer cells and omental adipocytes were reported [15], and a consistent finding was also shown in our study. In other words, we have shown that 
adipocytes contribute to the survival and growth of breast cancer cells through lipid transfer, lipolysis, and $\beta$-oxidation. Therefore, inhibiting the metabolic interaction between adipocytes and cancer cells is expected to hamper cancer growth. Among molecules involving the metabolic interaction, we focused on the role of FABP4. FABPs are $\sim 15 \mathrm{kDa}$ cytoplasmic proteins, which bind to endogenous fatty acids produced by lipolysis of adipocyte and transfer the FFA into the tumor cells [20]. In vitro data from this study showed that the co-culture of adipocytes and cancer cells leads to increased expression of FABP4 and the greatest expression was observed in TNBC type. When FABP4 in adipocytes was inhibited using FABP4 inhibitor (BMS309403), lipid transfer into cancer cells was significantly reduced, suggesting that FABP4 is the key molecule of metabolic interaction between adipocytes and breast cancer cells. In addition, lipolysis in adipocytes and $\beta$-oxidation of breast cancer cells were decreased, most markedly in TNBC. Therefore, FABP4 inhibition seems to effectively inhibit the interaction between adipocytes and breast cancer cells. Furthermore, when FABP4 was inhibited, cancer cell migration was also reduced in breast cancer cells, most significantly in TNBC. These results suggest that FABP4 is involved in tumor aggressiveness as well as metabolic interaction. In a previous study by Nieman et al. using a reverse phase protein array, a comparison of primary ovary cancer and omental metastatic tissue revealed that FABP4 protein expression was upregulated in omental metastatic tissue and inhibition of FABP4 markedly reduced adipocyte-mediated cancer invasion and lipid accumulation within cancer cells [15]. These findings suggest that FABP4 is a key molecule involved in the metabolic interaction between adipocytes and cancer cells.

In addition, IHC was conducted in human breast cancer tissues targeting lipid metabolism-related proteins between adipocytes and breast cancer cells. As a result, the expression of ACOX1, CPT1A, FABP4, and HSL showed a zonal distribution pattern. Furthermore, the expression of lipolysis-related proteins was examined by western blotting in the three different regions of breast cancer tissue, [central cancer with fibroblast (zone 1), cancer with adipocyte (zone 2 ), and adipocyte nearby cancer (zone 3)]. The expressions of PLIN1, HSL, and FABP4 were highest in zone 3, followed by zone 2 and 1, while CPT1A expression was highest in zone 2, followed by zone 3 and 1. It supports the metabolic interaction between adipocytes and cancer cells observed in our in vitro cell line study. Previous studies examined the expression of lipid metabolism-related proteins in breast cancer [21,22], but until recently, the expression in the adipose stroma was not examined.

The clinical significance of this study is that we suggested FABP4 inhibition as the potential therapeutic target in breast cancer. FABP4 inhibitor was first introduced as a therapeutic agent for type 2 diabetes [20]. The potency of the FABP4 inhibitor was previously verified in several studies [19, 23]. We used a well-known FABP4 inhibitor, BMS309403, a cell-permeable and potent inhibitor of FABP4, which targets the fatty acid-binding pocket [24]. BMS309403 markedly reduced lipid transfer, lipolysis of adipocytes, and $\beta$-oxidation of cancer cells in this study. Therefore, FABP4 inhibitor can be suggested as a new therapeutic agent to inhibit cancer growth. However, utilizing FABP4-defective cells would provide a better understanding regarding the role of FABP4 in energy transfer from adipocytes to cancer cells. Moreover, verification of the results from our study in a xenograft model and further clinical trials are required for its clinical application in breast cancer patients.

Taken together, in this study, we found that the metabolic interaction between adipocytes and breast cancer cells was present. Breast cancer cells increase the lipolysis in adipocytes and produce fatty acid, and fatty acid enters cancer cells. Also, adipocytes contribute to the survival and growth of cancer cells through increased mitochondrial $\beta$-oxidation by using fatty acid from adipocytes. The key molecule of the process is FABP4, which is combined with FFA and supports the migration to cancer cells. When FABP4 was suppressed, the metabolic interaction is reduced, suggesting its role as a potential therapeutic target.

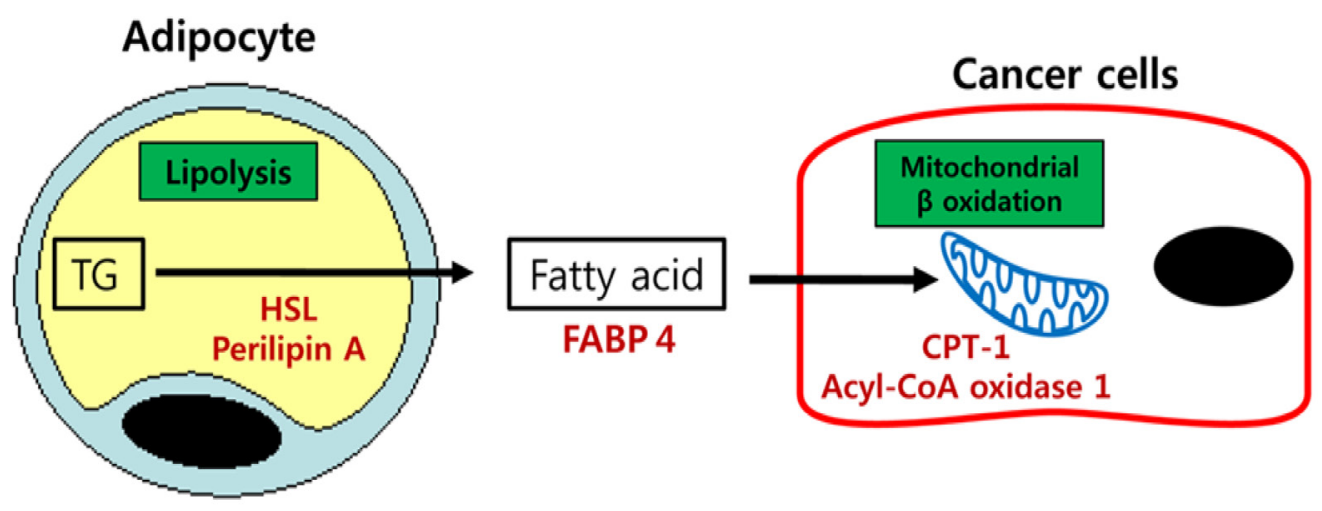

Figure 6. A schematic figure summarizing cell signaling pathways and energy transfer from adipocytes to cancer cells in breast cancer. 
Supplementary information is available in the online version of the paper.

Acknowledgments: This study was supported by a grant from National R\&D Program for Cancer Control, Ministry of Health \& Welfare, Republic of Korea (1420080). This study was supported by a faculty research grant from Yonsei University College of Medicine for 2014 (6-2014-0131).

\section{References}

[1] SABATIER R, FINETTI P, MAMESSIER E, ADELAIDE J, CHAFFANET $M$ et al. Prognostic and predictive value of PDL1 expression in breast cancer. Oncotarget 2015; 6: 54495464. https://doi.org/10.18632/oncotarget.3216

[2] ELSAMANY S, ABDULLAH S. Triple-negative breast cancer: future prospects in diagnosis and management. Med Oncol 2014; 31: 834. https://doi.org/10.1007/s12032-013-0834-y

[3] VANDEWEYER E, HERTENS D. Quantification of glands and fat in breast tissue: an experimental determination. Ann Anat 2002; 184: 181-184. https://doi.org/10.1016/s09409602(02)80016-4

[4] CALLE EE, KAAKS R. Overweight, obesity and cancer: epidemiological evidence and proposed mechanisms. Nat Rev Cancer 2004; 4: 579-591. https://doi.org/10.1038/nrc1408

[5] NIEMAN KM, ROMERO IL, VAN HOUTEN B, LENGYEL E. Adipose tissue and adipocytes support tumorigenesis and metastasis. Biochim Biophys Acta 2013; 1831: 1533-1541. https://doi.org/10.1016/j.bbalip.2013.02.010

[6] CORSA CA, BRENOT A, GRITHER WR, VAN HOVE S, LOZA AJ et al. The Action of Discoidin Domain Receptor 2 in Basal Tumor Cells and Stromal Cancer-Associated Fibroblasts Is Critical for Breast Cancer Metastasis. Cell Rep 2016; 15: 2510-2523. https://doi.org/10.1016/j.celrep.2016.05.033

[7] BUCHSBAUM RJ, OH SY. Breast Cancer-Associated Fibroblasts: Where We Are and Where We Need to Go. Cancers (Basel) 2016; 8. https://doi.org/10.3390/cancers8020019

[8] QIAO A, GU F, GUO X, ZHANG X, FU L. Breast cancer-associated fibroblasts: their roles in tumor initiation, progression and clinical applications. Front Med 2016; 10: 33-40. https://doi.org/10.1007/s11684-016-0431-5

[9] JUNG YY, KIM HM, KOO JS. The role of cancer-associated fibroblasts in breast cancer pathobiology. Histol Histopathol 2016; 31: 371-378. https://doi.org/10.14670/hh-11-700

[10] WARBURG O. On the origin of cancer cells. Science 1956; 123: 309-314

[11] MARTINEZ-OUTSCHOORN UE, BALLIET RM, RIVADENEIRA DB, CHIAVARINA B, PAVLIDES S et al. Oxidative stress in cancer associated fibroblasts drives tumor-stroma co-evolution: A new paradigm for understanding tumor metabolism, the field effect and genomic instability in cancer cells. Cell Cycle 2010; 9: 3256-3276. https://doi.org/10.4161/ cc.9.16.12553

[12] PAVLIDES S, TSIRIGOS A, VERA I, FLOMENBERG N, FRANK PG et al. Loss of stromal caveolin-1 leads to oxidative stress, mimics hypoxia and drives inflammation in the tumor microenvironment, conferring the "reverse Warburg effect": a transcriptional informatics analysis with validation. Cell Cycle 2010; 9: 2201-2219. https://doi.org/10.4161/cc.9.11.11848
[13] PAVLIDES S, WHITAKER-MENEZES D, CASTELLOCROS R, FLOMENBERG N, WITKIEWICZ AK et al. The reverse Warburg effect: aerobic glycolysis in cancer associated fibroblasts and the tumor stroma. Cell Cycle 2009; 8: 3984-4001. https://doi.org/10.4161/cc.8.23.10238

[14] BIFULCO M, PISANTI S. "Adiponcosis": a new term to name the obesity and cancer link. J Clin Endocrinol Metab 2013; 98: 4664-4665. https://doi.org/10.1210/jc.2013-2645

[15] NIEMAN KM, KENNY HA, PENICKA CV, LADANYI A, BUELL-GUTBROD R et al. Adipocytes promote ovarian cancer metastasis and provide energy for rapid tumor growth. Nat Med 2011; 17: 1498-1503. https://doi. org/10.1038/nm.2492

[16] IYENGAR P, COMBS TP, SHAH SJ, GOUON-EVANS V, POLLARD JW et al. Adipocyte-secreted factors synergistically promote mammary tumorigenesis through induction of anti-apoptotic transcriptional programs and proto-oncogene stabilization. Oncogene 2003; 22: 6408-6423. https:// doi.org/10.1038/sj.onc.1206737

[17] D'ESPOSITO V, PASSARETTI F, HAMMARSTEDT A, LIGUORO D, TERRACCIANO D et al. Adipocyte-released insulin-like growth factor-1 is regulated by glucose and fatty acids and controls breast cancer cell growth in vitro. Diabetologia 2012; 55: 2811-2822. https://doi.org/10.1007/ s00125-012-2629-7

[18] LIN Y, BERG AH, IYENGAR P, LAM TK, GIACCA A et al. The hyperglycemia-induced inflammatory response in adipocytes: the role of reactive oxygen species. J Biol Chem 2005; 280: 4617-4626. https://doi.org/10.1074/jbc.M411863200

[19] WANG Y, LIN HQ, LAW WK, LIANG WC, ZHANG JF et al. Pimozide, a novel fatty acid binding protein 4 inhibitor, promotes adipogenesis of 3T3-L1 cells by activating PPARgamma. ACS Chem Neurosci 2015; 6: 211-218. https://doi. org/10.1021/cn5002107

[20] LEHMANN F, HAILE S, AXEN E, MEDINA C, UPPENBERG J et al. Discovery of inhibitors of human adipocyte fatty acid-binding protein, a potential type 2 diabetes target. Bioorg Med Chem Lett 2004; 14: 4445-4448. https://doi. org/10.1016/j.bmcl.2004.06.057

[21] KIM S, LEE Y, KOO JS. Differential expression of lipid metabolism-related proteins in different breast cancer subtypes. PLoS One 2015; 10: e0119473. https://doi.org/10.1371/journal.pone.0119473

[22] JUNG YY, KIM HM, KOO JS. Expression of Lipid Metabolism-Related Proteins in Metastatic Breast Cancer. PLoS One 2015; 10: e0137204. https://doi.org/10.1371/journal. pone. 0137204

[23] SUHRE K, ROMISCH-MARGL W, DE ANGELIS MH, ADAMSKI J, LUIPPOLD G et al. Identification of a potential biomarker for FABP4 inhibition: the power of lipidomics in preclinical drug testing. J Biomol Screen 2011; 16: 467-475. https://doi.org/10.1177/1087057111402200

[24] SULSKY R, MAGNIN DR, HUANG Y, SIMPKINS L, TAUNK $P$ et al. Potent and selective biphenyl azole inhibitors of adipocyte fatty acid binding protein (aFABP). Bioorg Med Chem Lett 2007; 17: 3511-3515. https://doi.org/10.1016/j. bmcl.2006.12.044 